\title{
Detection of Human Vital Signs and Estimation of Direction of Arrival Using Multiple Doppler Radars
}

\author{
Yong-Jun $\mathrm{An}^{1} \cdot$ Byung-Jun Jang ${ }^{2} \cdot$ Jong-Gwan Yook ${ }^{1}$
}

\begin{abstract}
This paper presents a non-contact measurement method of vital signal by the use of multiple-input multiple-output (MIMO) bio-radar system, configured with two antennas that are separated by a certain distance. The direction of arrival (DOA) estimation algorithm for coherent sources was applied to detect vital signals coming from different spatial angles. The proposed MIMO bio-radar system was composed of two identical transceivers sharing single VCO with a PLL. In order to verify the performance of the system, the DOA estimation experiment was completed with respect to the human target at angles varying between $-50^{\circ}$ and $50^{\circ}$ where the bio-radar system was placed at distances (corresponding to $50 \mathrm{~cm}$ and $95 \mathrm{~cm}$ ) in front of a human target. The proposed MIMO bio-radar system can successfully find the direction of a human target.
\end{abstract}

Key words : Bio Radar, Doppler Radar, DOA, Heartbeat, MIMO, Respiration.

\section{Introduction}

Recently, remote sensing techniques have been actively studied as a means of measuring vital signs. Among these techniques, the Doppler radar sensing of vital signs has received substantial attention due to its simple system architecture and non-contact detection capability [1, 2]. Various Doppler radar architectures for sensing human vital signs have consequently been developed: a Doppler radar system based on board-level and chip-level design [3], a dual-antenna Doppler radar system compensated for background motion [4], a barrier penetration consisted of ultra-wide band (UWB), and a CW Doppler radar [5] are some examples. MIMO signal processing methods, such as blind source separation (BSS) and analytical constant modulus algorithm (ACMA), have attracted a great deal of interest for non-contact vital sign detection [6, 7]. Until recently, however, the existing MIMO techniques for vital sign detection have been mainly investigated to separate vital signs from multiple sources.

The use of MIMO radar technique for vital signal sensing has a number of potential advantages. For a given system design choice, some of these advantages are enhancement of the target detection performance, improvement of the angle estimation accuracy, and a decrease in the minimum detection velocity [8]. A MIMO bio-radar system can also be combined with well-known direction of arrival (DOA) estimation algorithms to provide solutions for direction finding of human subjects. In the present paper, a new approach is presented to find the direction of human vital signals with two identical transceiver architectures sharing a single VCO with a PLL. The MIMO Doppler radar system can be effectively applied to find a human target with respect to azimuth angle, making it a good candidate for applications in home health care systems, military surveillance, as well as security services. To the best of authors' knowledge, this is the first successfully implemented direction finding system for human targets based on a MIMO radar concept.

\section{II . MIMO Bio-Radar System Configuration}

The configuration of the proposed integrated MIMO bioradar system is shown in Fig. 1. This monostatic MIMO radar consists of two antennas and each antenna is used for both transmitting and receiving operations. Dual-transmitter topology provides a transmission of correlated signals, which is effective for obtaining independent phase information and improved detection accuracy of the Doppler motion of targets. When two signals arrive at antennas on the same circuit board, the signal to each antenna arrives at a different time due to the spatial path difference. This time delay introduces phase difference between two antennas. When the distance of separation

Manuscript received October 1, 2010 ; revised November 19, 2010. (ID No. 20101001-11J)

${ }^{1}$ Department of Electrical and Electronic Engineering, Yonsei University, Seoul, Korea

${ }^{2}$ Department of Electronics Engineering, Kookmin University, Seoul, Korea

Corresponding Author : Jong-Gwan Yook (e-mail : jgyook@yonsei.ac.kr) 


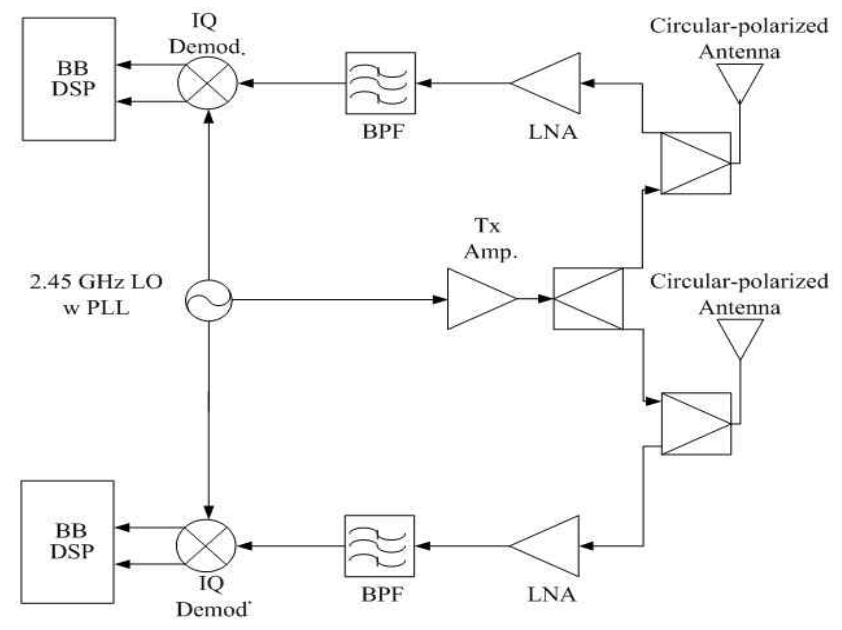

Fig. 1. Block diagram of the proposed MIMO bio-radar system.

between antennas is known, this makes it possible to estimate the direction of a human target by analyzing the phase difference data with the separation between antennas. For example, when the reflected signals from a human target received at two receivers contain same phase characteristics, the received signals experienced identical Doppler shifts from human vital signs. However, in practice, the signals undergo a time delay and the phase differences between receivers reflect the effects of the separation distance ( $d$ in Fig. 3), difference in incident angles, and the spacing between receivers. As formulated in Eq. (1), assuming R»d in Fig. 3, the received signals at spatially separated antennas contain information about the periodic movement $x(t)$, in addition to different phase terms from direction of target and space of antennas. To extract the direction of arrival from Eq. (1), two received signals are compared and can be expressed in the form of Eq. (2). When $\theta_{\text {track }}$ is equal to the DOA, the received signal (corresponding to $\mathrm{Rx}_{1}$ ) at one receiver and the received signal (corresponding to $\mathrm{Rx}_{2}$ ) at the other receiver can be considered as identical. Using the maximal ratio combining (MRC) method, the signals from both receivers are combined. This results in a two times higher SNR when $\theta_{\text {track }}$ is properly determined. The $\theta_{\text {track }}$ means also estimates the direction of the target.

$$
\begin{aligned}
S_{r x_{-} 1}=B_{1} \exp j\left(\frac{4 \pi x(t)}{\lambda}+\frac{\pi d}{\lambda} \sin \theta\right) \\
S_{r x_{-} 2}=B_{2} \exp j\left(\frac{4 \pi x(t)}{\lambda}-\frac{\pi d}{\lambda} \sin \theta\right) \\
S_{r x_{-} 2} \cdot \exp j\left(\frac{2 \pi d}{\lambda} \sin \theta_{\text {track }}\right)=B_{2} \exp j\left(\frac{4 \pi x(t)}{\lambda}-\frac{\pi d}{\lambda} \sin \theta\right) \\
\cdot \exp j\left(\frac{2 \pi d}{\lambda} \sin \theta_{\text {track }}\right) \\
\approx S_{r x_{-} 1}
\end{aligned}
$$

The ratio between the received signal power at $\mathrm{RX}_{1}$ to the power at $\mathrm{RX}_{2}$ corresponds to the phase difference between two receivers:

$$
\begin{aligned}
S_{r x_{-} 2} / S_{r x_{-} 1} & =\frac{B_{2}}{B_{1}} \frac{\exp j\left(\frac{4 \pi x(t)}{\lambda}-\frac{\pi d}{\lambda} \sin \theta\right)}{\exp j\left(\frac{4 \pi x(t)}{\lambda}+\frac{\pi d}{\lambda} \sin \theta\right)} \\
& \approx \exp j\left(-\frac{2 \pi d}{\lambda} \sin \theta_{\text {track }}\right)
\end{aligned}
$$

\section{2-1 System Configuration}

Previously, we have demonstrated $2.4 \mathrm{GHz}$ bio-radar systems [9, 10] with improved noise performance using PLL [11]. We have also introduced a circular polarized antenna to reduce size and to allow use of a single antenna for bio-radar applications $[12,13]$. In the present paper, a MIMO bio-radar system is introduced that consists of two bio-radar system sharing identical VCO with a PLL and new concept of direction finding of human target based on phase difference between two receivers is presented. Fig. 2 shows a photograph of the implemented MIMO bio-radar system. The antenna structure consists of two identical annular ring radiating elements with their centers separated by a distance of 0.75 $\lambda$ (where $\lambda$ is the wavelength). Each radiating element is fed by $90^{\circ}$ hybrid couplers, as shown in Fig. 2(a). The measured isolation between the $\mathrm{Tx}$ and $\mathrm{Rx}$ is better than $20 \mathrm{~dB}$. The isolation between antennal and 2 is also better than $25 \mathrm{~dB}$ in HFSS simulation. The phase locked LO signal is split, amplified, transferred to the down-conversion mixer, and delivered to the antennas. A single VCO with a PLL also provides two signals of identical frequency, one for the $\mathrm{Tx}$ and the other for mixer driving. The output power level of the proposed bio-radar system is set to $0 \mathrm{dBm}$ with an antenna gain of $0 \mathrm{dBi}$. The antenna has right-hand circular polarization (RHCP) at transmitting mode and left-hand circular polarization (LHCP) at receiving mode. Due to the symmetry and inverted antenna array structure, signals have $180^{\circ}$ phase difference, so the null radiation pattern is represented at $0^{\circ}$. Mathematically each quadrature receiver down-converts the received signal into a complex number: its real part is the in-phase component or I signal, while its imaginary part is the quadrature component or Q signal.

\section{Experiment and Result}

The operation of the proposed MIMO bio-radar system is demonstrated by measuring human vital signs. A typical measurement is shown in Fig. 4. The data are 


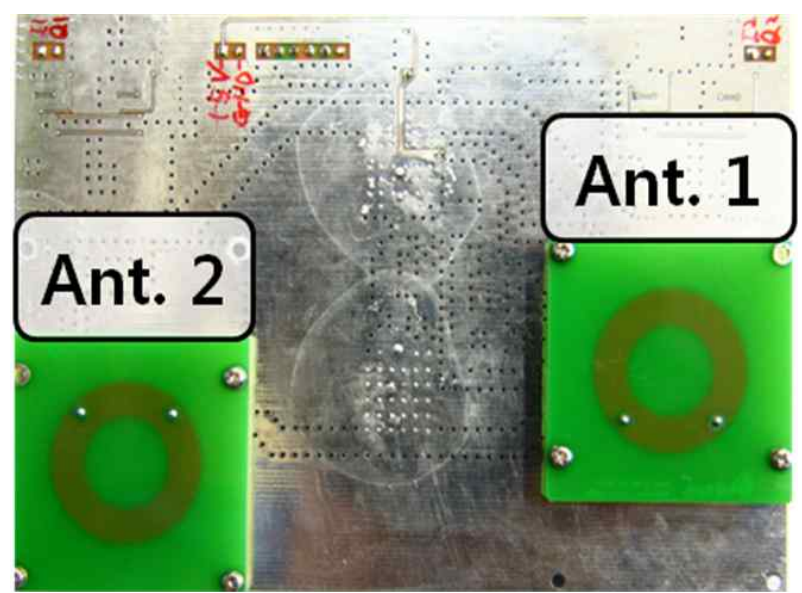

(a) Antennas

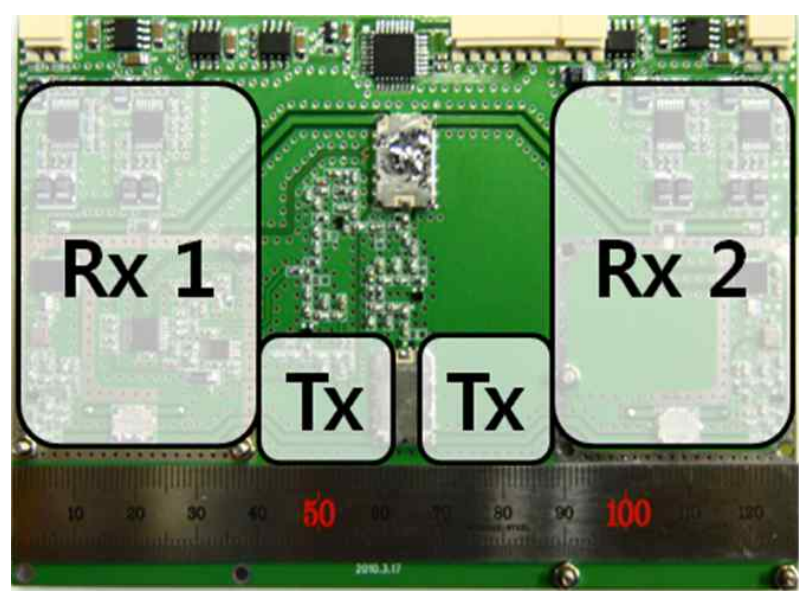

(b) Active circuits

Fig. 2. Photograph of the MIMO bio-radar system.

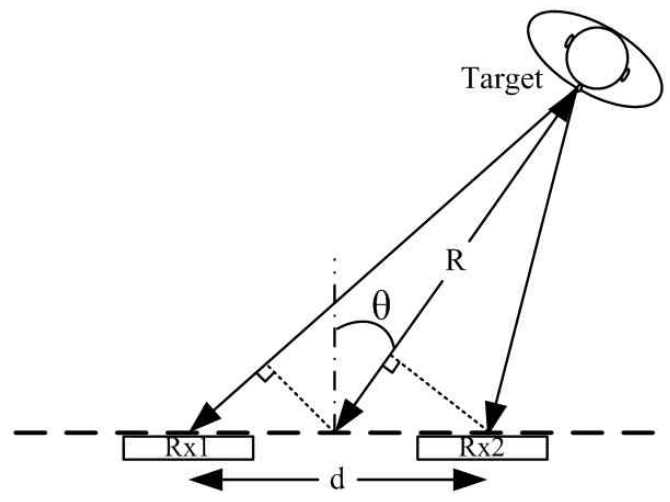

Fig. 3. Description of phase differences between $\mathrm{Rx}_{1}$ and $\mathrm{Rx}_{2}$.

collected in response to the trajectories of human target movement between $-50^{\circ}$ to $50^{\circ}$ (where $0^{\circ}$ corresponds to a human target facing the MIMO bio-radar system) at a fixed distance of $0.5 \mathrm{~m}$. The experimentally collected data are transferred to a computer via a data acquisition board (NI-DAQ) and a digital signal processing technique for Doppler radar sensing of vital signs

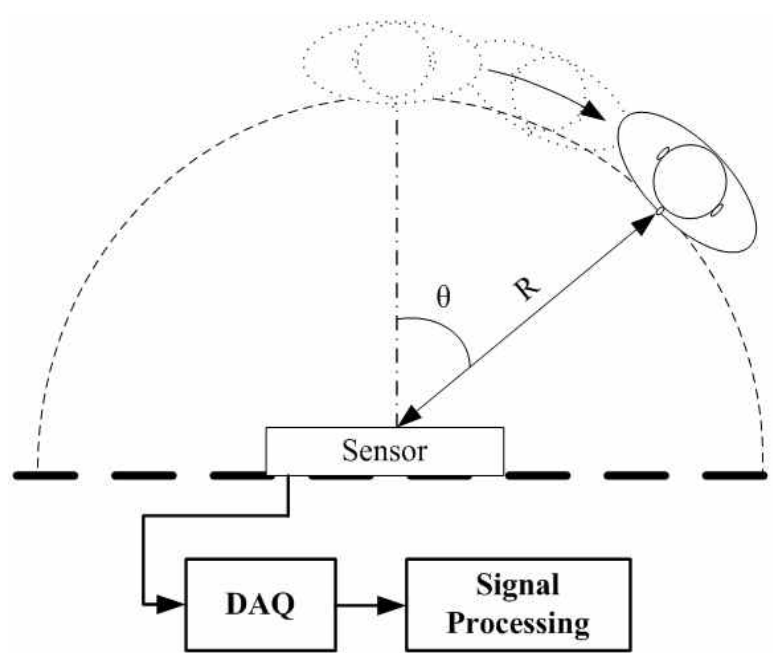

Fig. 4. Appearance of the measurement setup.

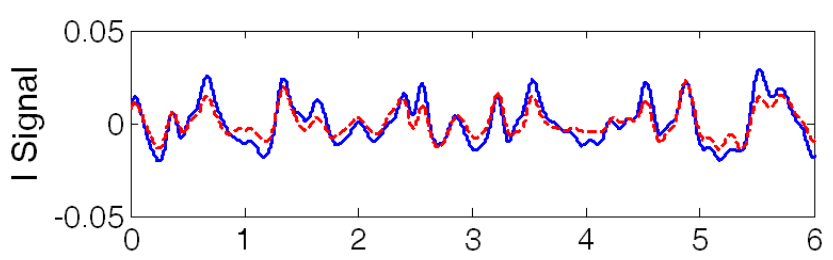

(a) I signal

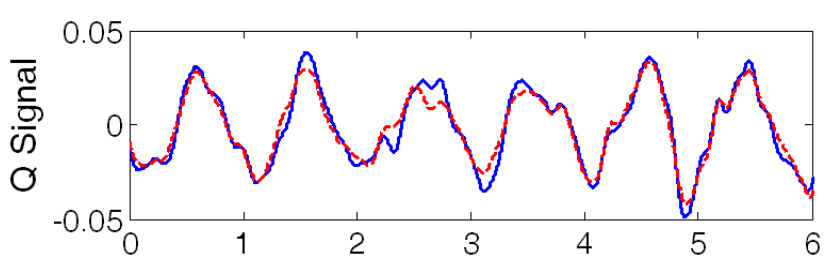

(b) Q signal

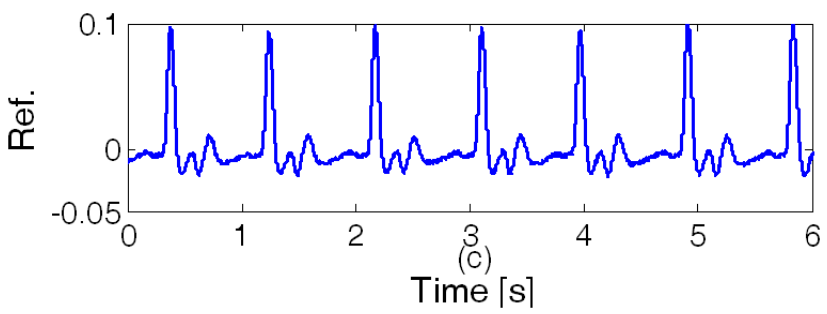

(c) Reference heartbeat signal

Fig. 5. Measured signals for targets at a distance of $50 \mathrm{~cm}$ and at a direction of $30^{\circ}$ under breathing conditions: bandpass filtered heartbeat signal (solid line: $\mathrm{Rx}_{1}$ signal, dotted line: converted $\mathrm{Rx}_{2}$ signal).

is implemented. The I and Q channel output signals received at $\mathrm{Rx}_{1}$ and $\mathrm{Rx}_{2}$ are different because of the phase difference between $\mathrm{Rx}_{1}$ and $\mathrm{Rx}_{2}$, arising from the relative distance from the antennas to the target. The $\theta_{\text {track }}$ can then be determined from eq (3). As shown in Fig. 5, the $\mathrm{I}$ and $\mathrm{Q}$ channel output signals received at $\mathrm{Rx}_{2}$ are approximately the same as $\mathrm{I}_{1}$ and $\mathrm{Q}_{1}$ of the $\mathrm{Rx}_{1}$. 


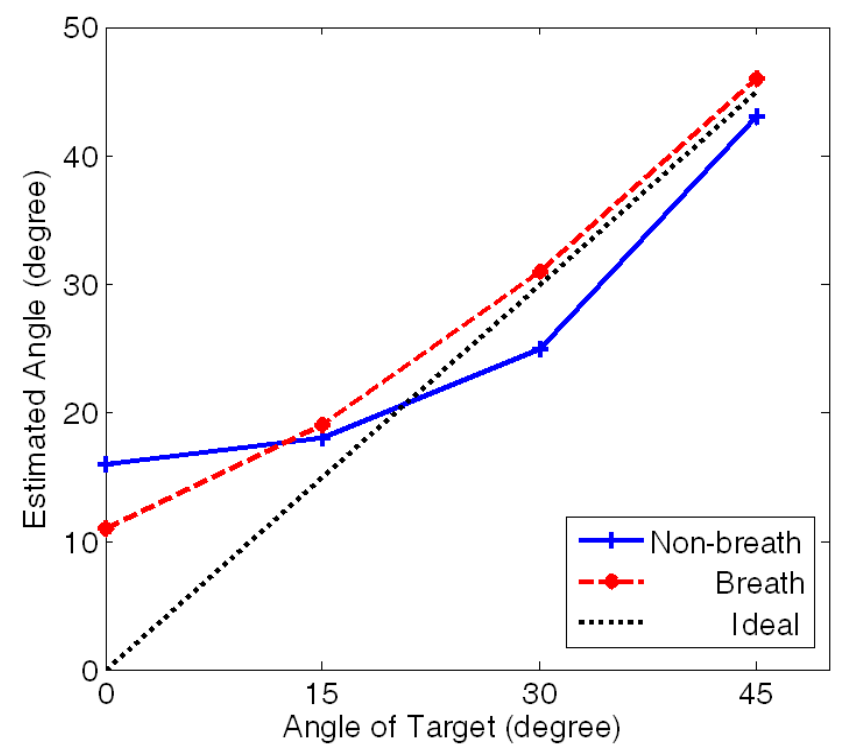

Fig. 6. Measured results of DOA estimation at the distance of $50 \mathrm{~cm}$.

This result is not unexpected because the output signals are measured from the same human target. It is worth noting that $3-\mathrm{dB}$ diversity gain is achieved by transmitting the same signals from two spatially separated antennas.

The measured data with respect to various incident angles $\left(15^{\circ}, 30^{\circ}\right.$, and $\left.45^{\circ}\right)$ are displayed in Fig. 6 and show reasonably good performance for DOA estimation. Two cases are presented: non-breathing and breathing cases. Non-breathing means that the target human hold his breath, while breathing means the target human breathes normally (in other words, this is respiration). These results indicate that the MIMO bio-radar system is suitable for finding the direction of a human target based on the concept of phase differences between $\mathrm{Rx}_{1}$ and $\mathrm{Rx}_{2}$. Fig. 7 and Fig. 8 show the measured heartbeat signals when the subject is fixed at $95 \mathrm{~cm}$ from the antennas. Note that there is slight signal quality due to increased range loss. Experiments have also done from $0^{\circ}$ up to $45^{\circ}$. DOA estimation results are summarized in Fig. 8 and reveal excellent performance.

It should be noted that since a two-antenna array has a null at the angle of $0^{\circ}$ (where there is a direct line of sight between the human target and MIMO bio-radar system), for the case of both breathing and non-breathing conditions, the DOA estimation produces a less accurate result at the angle of $0^{\circ}$. This problem can be solved if the transmitting signals are processed for the non-coherent regime. In general, the DOA estimation error for the breathing case is smaller than that of the non-breathing case, because of the larger SNR for the breathing case.
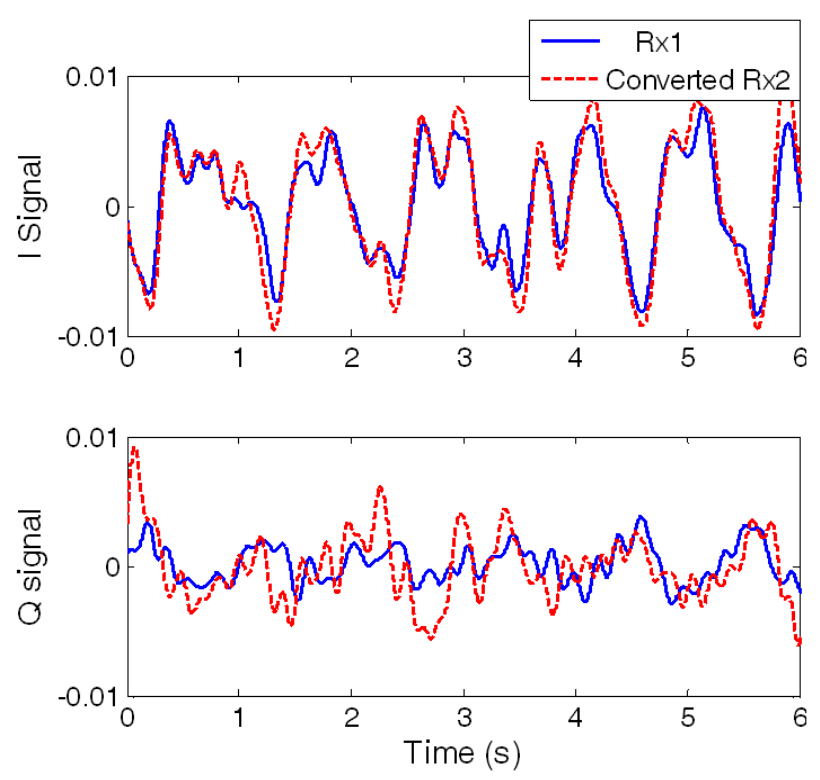

Fig. 7. Measured I and Q channel signals at the distance of $95 \mathrm{~cm}$ with the direction of $25^{\circ}$ under the breathing condition (solid line: $\mathrm{Rx}_{1}$ signal, dotted line: converted $\mathrm{Rx}_{2}$ signal).

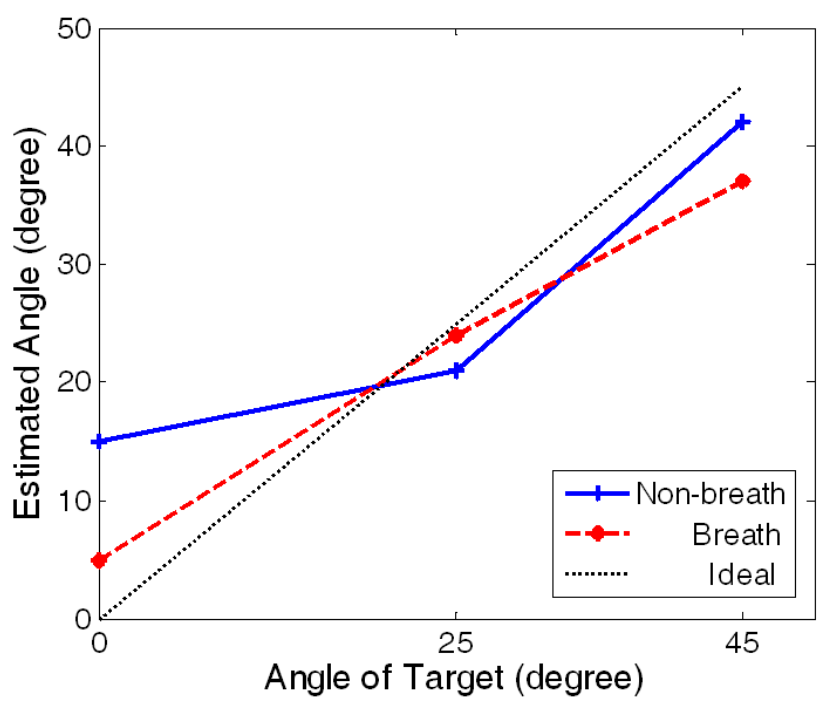

Fig. 8. Measured results of DOA estimation at the distance of $95 \mathrm{~cm}$.

\section{Conclusions}

A new multiple-input multiple-output (MIMO) bioradar system is proposed for non-contact measurement of human vital signs. The proposed MIMO bio-system consists of two identical transceiver architectures sharing a single VCO with a PLL. In particular, the phase information of the MIMO bio-radar provides useful information for identifying the direction of a human target. In the respiration case, this bio-radar system shows good 
sensing of heartbeats and position angle of the human target. To verify the vital sign monitoring performance, a DOA estimation experiment has been completed with respect to the human target at angles varying between $-50^{\circ}$ and $50^{\circ}$ at the distance of both $50 \mathrm{~cm}$ and $95 \mathrm{~cm}$.

This research was supported by the MKE (The Ministry of Knowledge Economy), Korea, under the ITRC (Information Technology Research Center) support program supervised by the NIPA(National IT Industry Promotion Agency)" (NIPA-2010-(C1090-1021-0005)).

\section{References}

[1] K. M. Chen, D. Misra, H. Wang, H. R. Chuang, and E. Postow, "An X-band microwave life-detection system," IEEE Trans. Biomed. Eng., vol. 33, pp. 697702, Jul. 1986.

[2] S. G. Kim, H. Kim, Y. S. Lee, I. S. Kho, and J. G. Yook, "5.8 GHz vital signal sensing Doppler radar using isolation-improved branch-line coupler," Radar Conference, EuRAD 2006, 3rd European, pp. 249252, Sep. 2006.

[3] Li Changzhi, J. Cummings, J. Lam, E. Graves, and $\mathrm{Wu}$ Wenhsing, "Radar remote monitoring of vital signs," Microwave Magazine, IEEE, vol. 10, no. 1, pp. 47-56, Feb. 2009.

[4] Fletcher, R. Jing Han, "Low-cost differential frontend for Doppler radar vital sign monitoring," Microwave Symposium Digest, MTT '09, IEEE MTT- S International, pp. 1325-1328, Jun. 2009.

[5] V. M. Lubecke, O. Boric-Lubecke, A. Host-Madsen, and A. E. Fathy, "Through-the-wall radar life detection and monitoring," Microwave Symposium, IEEE/ MTT-S International, pp. 769-772, 3-8, Jun. 2007.

[6] Alexander Vergara, Nicolas Petrochilos, Olga BoricLubecke, Anders Host-Madsen, and Victor Lubecke, "Blind source separation of human body motion using direct conversion Doppler radar," Microwave Symposium Digest, 2008 IEEE MTT-S International, pp. 1321-1324, Jun. 2008.

[7] D. Smardzija, O. Boric-Lubecke, A. Host-Madsen, V. M. Lubecke, T. II. Sizer, A. D. Droitcour, and G. T. A. Kovacs, "Applications of MIMO techniques to sensing of cardiopulmonary activity," Wireless Communications and Applied Computational Electromagnetics, IEEE/ACES International Conference on, pp. 618-621, Apr. 2005.

[8] Jian Li, Peter Stoica, MIMO Radar Signal Processing, Wiley, 2008.

[9] B. J. Jang, S. H. Wi, J. G. Yook, M. Q. Lee, and K. J. Lee, "Wireless bio-radar sensor for heartbeat and respiration detection," Progress in Electromagnetic Research (PIER) C, vol. 5, pp. 149-168, 2008.

[10] Y. J. Lee, B. J. Jang, and J. G. Yook, "An 2.4 $\mathrm{GHz}$ bio-radar system for non-contact measurement of heart and respiration," The Journal of Korea Electromagnetic Engineering Society, KEES, vol. 19, pp. 191-199, 2008.

[11] B. J. Jang, J. G. Yook, W. Na, and M. Q. L., "Noise analysis and measurement for a CW bio-radar system for non-contact measurement of heart and respiration rate," The Journal of Korea Electromagnetic Engineering Society, KEES, vol. 19, pp. 1010-1019, 2008.

[12] S. S. Myoung, B. J. Jang, J. H. Park, and J. G. Yook, "2.4 GHz bio-radar system with improved performance by using phase-locked loop," Microwave and Optical Technology Letters, KEES, vol. 52, no. 9, pp. 2074-2076, Sep. 2010.

[13] B. J. Jang, J. H. Park, J. G. Yook, J. H. Moon, and K. J. L., "A $2.4 \mathrm{GHz}$ bio-radar system with small size and improved noise performance using single circular-polarized antenna and PLL," The Journal of Korea Electromagnetic Engineering Society, KEES, vol. 20, pp. 1325-1332, 2009. 


\section{Yong-Jun An}

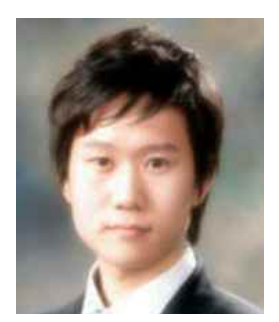

was born in Seoul, Korea, in 1986. He received the B.S. degree from Yonsei University, Seoul, Korea, in 2009, and currently working toward the Ph.D. degree in electrical engineering at Yonsei University. His research interests include RF system, antenna, and RF Integrate circuits.

\section{Byung-Jun Jang}

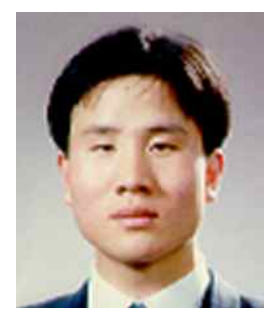

(S'90-M'96) received the B.S., M.S., and $\mathrm{Ph} . \mathrm{D}$. degrees in electronic engineering from Yonsei University, Seoul, Korea, in 1990, 1992, and 1997, respectively. From 1995 to 1999 , he was with LG Electronics, Seoul, where he developed code-division multipleaccess and digital enhanced cordless telecommunication RF modules. From 1999 to 2005, he was with the Electronics and Telecommunications Research Institute, Daejeon, Korea, where he performed research in the fields of satellite RF components and monolithic microwave integrated circuits. In 2005, he joined Kookmin University, Seoul, where he is currently with the Department of Electrical Engineering. He is currently interested in the areas of RF circuit design and system analysis, radio-frequency-identification interference modeling, and biosensor design.

\section{Jong-Gwan Yook}

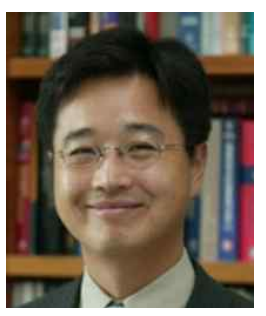

(S'89-M'97) was born in Seoul, Korea. He received the B.S. and M.S. degrees in electronics engineering from Yonsei University, Seoul, Korea, in 1987 and 1989, respectively, and the Ph.D. degree from The University of Michigan, Ann Arbor, MI, in 1996. He is currently a Professor with the School of Electrical and Electronic Engineering, Yonsei University. His main research interests are in the areas of theoretical/numerical electromagnetic modeling and characterization of microwave/millimeter-wave circuits and components, design of radio frequency integrated circuits (RFIC) and monolithic microwave integrated-circuit (MMIC), analysis and optimization of high-frequency highspeed interconnects, including RF microelectromechanical systems (MEMS), based on frequency as well as time-domain fullwave methods, and development of numerical techniques. Recently, his research team is developing various biosensors, such as carbon-nano-tube RF biosensor for nanometer size antigen-antibody detection as well as remote wireless vital signal monitoring sensors. 